\title{
THE IMPACT OF MOSFET-BASED SENSORS *
}

\section{P BERGVELD}

Department of Electrical Engineering, Twente University of Technology, PO Box 217, 7500 AE Enschede (The Netherlands)

(Received May 21, 1985, in revised form October 4, 1985, accepted October 29, 1985)

\begin{abstract}
The basic structure as well as the physical existence of the MOS fieldeffect transistor is without doubt of great importance for the development of a whole series of sensors for the measurement of physical and chemical environmental parameters

The equation for the MOSFET drain current already shows a number of parameters that can be directly influenced by an external quantity, but small technological variations of the original MOSFET configuration also give rise to a large number of sensing properties All devices have in common that a surface charge is measured in a sllicon chıp, depending on an electric field in the adjacent insulator

FET-based sensors such as the GASFET, OGFET, ADFET, SAFET, CFT, PRESSFET, ISFET, CHEMFET, REFET, ENFET, IMFET, BIOFET, etc developed up to the present or those to be developed in the near future will be discussed in relation to the considerations mentioned above
\end{abstract}

\section{Introduction}

The measurement of semiconductor surface charge as a function of an electric field perpendicular to the surface was mentioned as early as 1925 by Lilienfeld and Heil as a possible principle for an electronic device that would not consume any power from the input source creating the electric field [1] In the mid-1940s Shockley and Pearson put the theory on a firm footing [2], but it took until the 1960s before these devices could actually be produced using the experience gained with silicon planar technology [3]

The most essential property of the MOS device that was created is its sensitivity, resulting from the fact that minority carriers are measured on

* Paper presented at the Third International Conference on Solid-State Sensors and Actuators (Transducers '85), Phıladelphı, PA, U S A , June 11 - 14, 1985 
the surface of a semiconductor with a majority doping of the opposite sign (n-channel in p-type substrate and vice versa) From an electronic viewpoint, the main advantage of the device is the extremely high inherent impedance transformation from input to output, which has a value of the order of $10^{11}$ This property also makes the MOS device very attractive for sensor applications, as will be outlined in the following sections

\section{The MOS transistor}

First we will briefly summarize the most important MOS equations and parameters for an $\mathrm{n}$-channel MOS transistor

In first-order MOS transistor theory, the equation for the drain current $I_{\mathrm{D}}$, in the unsaturated region $\left(V_{\mathrm{D}}<V_{\mathrm{G}}-V_{\mathrm{T}}\right)$ is

$I_{\mathrm{D}}=\mu C_{\mathrm{ox}} \frac{W}{L}\left[\left(V_{\mathrm{G}}-V_{\mathrm{T}}\right) V_{\mathrm{D}}-\frac{1}{2} V_{\mathrm{D}}^{2}\right]$

where $\mu$ is the electron mobulity in the channel, $C_{\text {ox }}$ the oxide capacitance per unit area, $W / L$ the channel width-to-length ratio, $V_{\mathrm{G}}$ and $V_{\mathrm{D}}$ the applied gate-source and drain-source voltages respectively and $V_{\mathbf{T}}$ is the threshold voltage, which can be described as follows

$V_{\mathrm{T}}=V_{\mathrm{FB}}-\frac{Q_{\mathrm{B}}}{C_{\mathrm{ox}}}+2 \phi_{\mathrm{f}}$

$Q_{\mathrm{B}}$ is the bulk depletion charge per unit area, $\phi_{\mathrm{f}}$ is the Fermi potential difference between the doped bulk silicon and intrinsic silicon and $V_{\mathrm{FB}}$ is the flatband voltage, which is given by

$V_{\mathrm{FB}}=\frac{\Phi_{\mathrm{M}}}{q}-\frac{\Phi_{\mathrm{si}}}{q}-\frac{Q_{1 \mathrm{t}}+Q_{\mathrm{f}}}{C_{\mathrm{ox}}}$

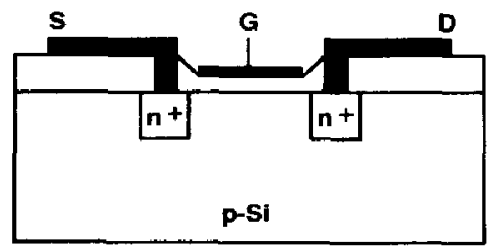

$I_{D}=\mu C_{o x} \frac{W}{L}\left[\left(V_{G}-V_{T}\right) V_{D}-1 / 2 V_{D}{ }^{2}\right]$

$v_{T=v_{F \theta}}-\frac{a_{B}}{c_{o x}}+2 \phi$,

$V_{F B}=\frac{\Phi_{M}}{q}-\frac{\Phi_{S_{1}}}{q}-\frac{a_{1 t}+Q_{f}}{C_{o x}}$

Fig 1 Schematic representation and basic operation of the MOSFET 
$\Phi_{\mathrm{M}}$ and $\Phi_{\mathrm{si}}$ are the metal and slicon work functions respectively, while $Q_{\mathrm{t} t}$ and $Q_{\mathfrak{f}}$ are the charge of the interface traps and the fixed oxide charge respectively, both per unit area

In the first instance the operation of a MOS device in the unsaturated region can be fully explained by these three equations, as summarized in Fig 1

In the saturated region eqns (2) and (3) are still valid, but eqn (1) has to be replaced by

$I_{\mathrm{D}}=\frac{1}{2} \mu C_{\mathrm{ux}} \frac{W}{L}\left(V_{\mathrm{G}}-V_{\mathrm{T}}\right)^{2}$

\section{The MOS transistor as a sensor}

In order to consider possible sensor properties of the MOS transistor, we have to investigate the MOS equation as described in the previous section with respect to environmental influences upon the distinguishable parameters

Applied as an electronic device, all parameters described in Section 2 are assumed to be constant, resulting in a well-defined relation between the output of the device, which is the drain current $I_{\mathrm{D}}$, and the input $V_{\mathrm{G}}$

It is well known, however, that the physical parameters $\mu$ and $\phi_{\mathrm{f}}$ are influenced by external conditions such as temperature, light and radiation These sources of interference should be disregarded in the case of an electronic application of the MOSFET, but on the contrary can be used for sensing functions of the device in the case of a temperature sensor, an optical sensor or a radiation sensor

These sensing functions are, however, not reserved for MOS devices, because the origin is a pure solid-state effect in the bulk of the semiconductor which can also, even more easily, be used to advantage with other semiconductor configurations such as simple diffused resistors, diodes and transistors Therefore no practical MOS-based sensors are avallable based on modulation of $\mu$ and $\phi_{\mathrm{f}}$, except for MOS infrared detectors [4]

A unique feature of a MOS device is, however, its dependence on the metal work function $\Phi_{M}$ It is known from MOS technology that the threshold voltage $V_{\mathrm{T}}$ depends on the kind of metal used for the gate In this way contact potentials $\phi_{\mathrm{ms}}=\left(\Phi_{\mathrm{M}} / q\right)-\left(\Phi_{\mathrm{s} 1} / q\right)$ could be made measurable for the first time in a direct way, such measurements previously being only possible by the vibrating reed method

It will be clear that, if the work function of the gate metal can be controlled by an external parameter, the MOS device is a direct sensor for this parameter In 1975 Lundstrom et al [5] showed that with a catalytically active gate metal (instead of the aluminium commonly used in MOS technology) such a situation can be created They found that with palladium (Pd) as the gate metal, a hydrogen-sensitive MOS device could be realızed 


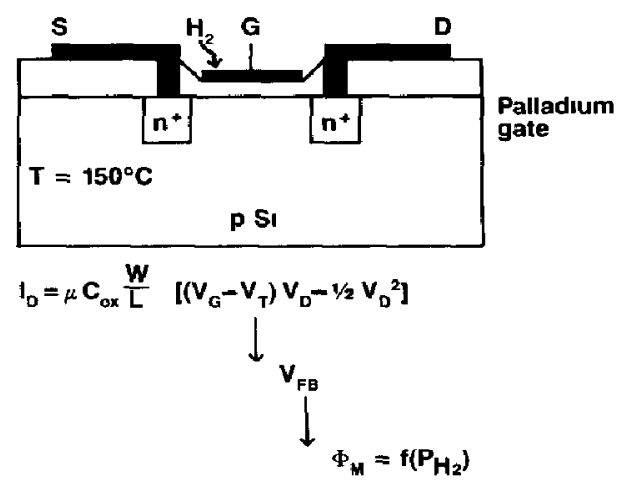

Fig 2 Schematic representation and basic operation of the GASFET

and that the chemical sensitivity of this structure can be properly described by a chemical control of the gate metal work function

The reason is that hydrogen in the ambient dissociatively adsorbs at the outer Pd surface, whlle the resulting hydrogen atoms diffuse through the thin $\mathrm{Pd}$ layer and adsorb at the $\mathrm{Pd} / \mathrm{SiO}_{2}$ interface Detalled analysis of this process results in a description of the metal work function as follows

$\frac{\Delta \Phi_{\mathrm{M}}}{q}=\left(\frac{\Delta \Phi_{\mathrm{M}_{\max }}}{q}\right)\left(\frac{K P_{\mathrm{H}_{2}}{ }^{1 / 2}}{1+K P_{\mathrm{H}_{2}}{ }^{1 / 2}}\right)$

where $K$ is an overall equilibrium constant, $P_{\mathrm{H}_{2}}$ the partial hydrogen pressure of the ambient and $\Delta \Phi_{M_{\max }}$ is the maximal shift in the metal work function which is obtained at total coverage with $\mathrm{H}$ atoms of the avalable number of adsorption sites per unit area at the $\mathrm{Pd} / \mathrm{S}_{1} \mathrm{O}_{2}$ interface

These devices are often operated at an elevated temperature (50 $150{ }^{\circ} \mathrm{C}$ ) in order to speed up the catalytıc reactions on the Pd surface This principle, which is basically the measurement of a contact potential and th.erefore explicitly supporting the MOSFET concept (see Fig 2), is in use not only for the measurement of hydrogen, but also for other gases such as $\mathrm{CO}$, in which case the $\mathrm{Pd}$ gate should be rather porous [6] It has also been shown that a large ammonia sensitivity can be obtained by using thin cataly tic metals as gates [7]

\section{Changes in the MOS transistor configuration for sensor functions}

Most of the MOSFET-based sensors function through small technological variations of the gate input They all have in common a silicon substrate with diffused source and drain regions, covered with a thin or thick oxide layer The different configurations can only be distinguished in the structures outside this oxide On the other hand, these various structures all have in common that they are the result of MOSFET-compatible technologies 
The most simple technological modification of the MOSFET configuration is the omission of the gate metal A technological variation that is sometimes more difficult is the use of a gate at a certain distance, this distance being bridged by a certain material The resulting sensors are sensitive to either a chemical or physical quantity that changes certain parameters of this added material

In the next sections the various FET-based sensors will be described along the lines of minor or major complicated additional structures of the compound gate areas

\section{The OGFET}

The Open Gate Field Effect Transistor (OGFET) was introduced by Johannessen in 1970 [8], who exposed a MOSFET, from which the gate metal was simply omitted, to a gaseous environment A strong variation of the drain current was observed as a function of the partial pressure of polar gases such as water vapour and methanol The experimental results were corroborated by Thorstensen, who also observed a response to non-polar gases All results obtained were qualitatively not affected by the oxide thickness, which varied from $2-50 \mathrm{~nm}$, but these oxides were always of very poor quality, allowing a certain amount of electron transport through the oxide of simultaneously fabricated MOSFETs The operation of the devices has not been satisfactorily investigated Only suggestions are given concerning the operational mechanism The gas adsorption might result in a dissociation of gas molecules and hence charged particles, which are possibly transported along the oxide surface under the influence of the electric fringe field resulting from the drain-source voltage The diffusion of charged and uncharged particles into the oxide towards the $\mathrm{S} 1 / \mathrm{SlO}_{2}$ interface may also play a certain role Further, a change in the dielectric constant of the oxide is suggested as being a cause of drain current modulation These various possibilities of operation are given schematically in Fig 3 The poor comprehension of OGFET operation is a serious drawback in the further development of this type of gas-sensitive FET sensor
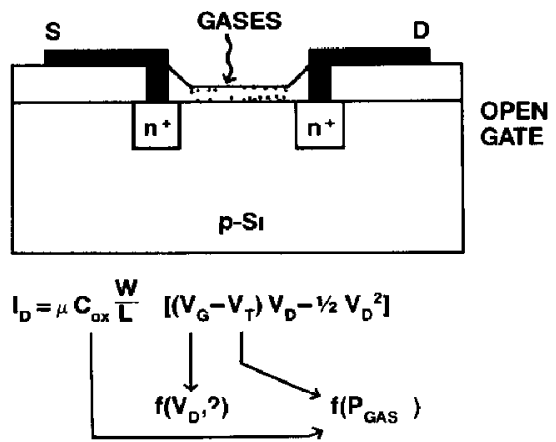

Fig 3 Schematic representation and basic operation of the OGFET 


\section{The ADFE'T}

A patented version of the OGFE'T described in the previous section is the Adsorption Field Effect Transistor (ADFET) [9] The difference is due to a limitation in the oxide thickness, which has a maximum of $50 \AA$ for the ADFET It is only for oxide thicknesses below this value that the ADFET appears to respond to all kinds of gases that have a permanent net dipole moment, such as $\mathrm{H}_{2} \mathrm{O}, \mathrm{NH}_{3}, \mathrm{HCl}, \mathrm{CO}, \mathrm{NO}, \mathrm{NO}_{2}$ and $\mathrm{SO}_{2}$ The explanation of the observed sensitivity is that the drain current is determined by the fringing field of the adsorbed molecules This may also account for the fact that devices with thicker oxides do not respond to adsorbed gases, as the externally sensible field of a dipole rapidly falls off in proportion to the distance $\left(1 / r^{3}\right)$ The device structure and operation are summarized in Fig 4

Selectivity of the devices is claimed to be realizable by proper chemical modification of the oxide surface, such as replacement of the silicon/oxygen bonds by sllicon/carbon bonds, which are in turn again modified with special groups For example, to enhance the response to $\mathrm{NO}$ and $\mathrm{NO}_{2}$ one should use silyl derivatives with amino or phenyl groups and for an $\mathrm{SO}_{2}$ sensor, silyl des ivatives with hydroxyl, amide or imidazole groups should be used In the ADFET patent a multisensor array has been claimed, each sensor having its own primary response to a certain gaseous component or class of components In this way the development of a monitoring system for characterizing the presence and concentration of selected substances in a gaseous environment is proposed

A serious problem concerning the application of the OGFET and the ADFET is the open-gate construction, which makes the devices very sensitive to all kinds of electrical interference sources that cause an electric field in the oxide A more suitable construction from a practical point of view will be obtained if a gate metal is added at a short distance Such a device is described in the next section

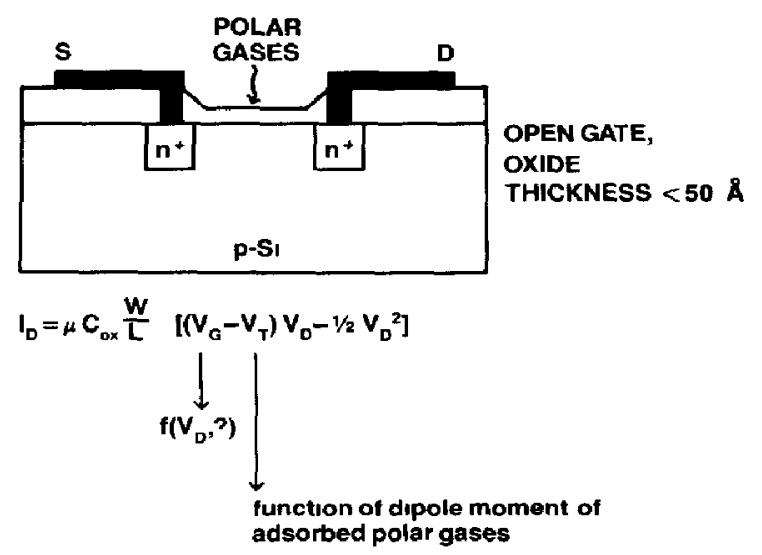

Fig 4 Schematic representation and basic operation of the ADFET 

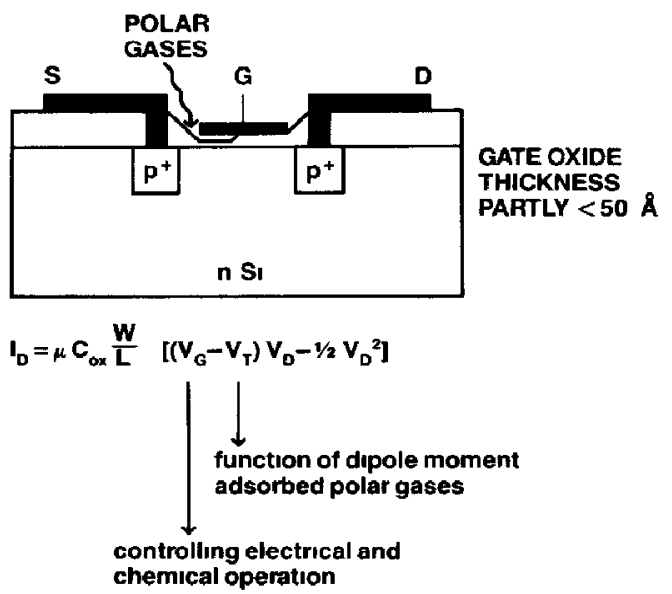

Fig 5 Schematic representation and basic operation of the SAFET

\section{The SAFET}

The Surface Accessible Field Effect Transistor, SAFET, is a modification of open-gate gas-sensing FETs Now the gate insulator consists partly of an arr gap, created by underetching of the polysilicon gate [10] In this way the gas has direct access to the silicon surface without loss of the electrostatic shielding action of the gate metal

As with the ADFET, the drain current of the SAFET appears to increase on being exposed to polar gases like water, acetone and alcohols Assuming that the underetched gate region is nevertheless covered with a native oxide, the operation mechanism will be the same as for the ADFET, but an increase of drain current is limited by the series channel resistance at the unetched part of the transistor This operation is schematically represented in Fig 5

The existence of the gate metal is not only favourable for diminishing the interference of external electrical sources, but also operates as an electronic input of the device This appears to be very useful for improvement of the reversibility of the adsorption process, the sensitivity and the stability of the device

The best results were obtained with an integration technique where the time to reach a preset value of the drain current is measured after applying a negative gate voltage This time appears to be a unique function of the concentration of the polar gas component to be detected After each measurement a short positive pulse is applied to the gate electrode before the next measurement is carried out

\section{The charge flow transistor, CFT}

The CFT construction, first published by Senturia et al [11], can be seen as a modification of the MOSFET, where the gate is mismatched in the sense that it does not cover the actual gate area but is placed beside or 

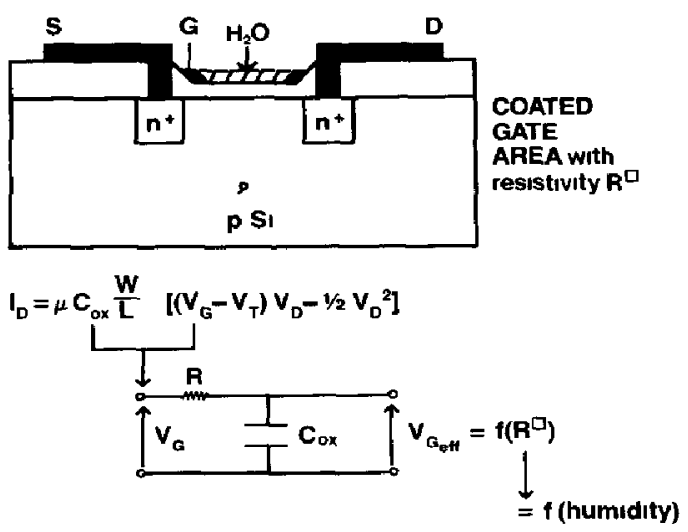

Fig 6 Schematic representation and basic operation of the CFT

around it The gate area itself is covered with a resistive material that is in contact with the laterally shifted gate As a result there will be a time delay between the onset of a voltage step between the eccentric gate and the source and the appearance of a complete channel This time delay depends, amongst other things, on the resistivity of the thin resistive film, which determines the time constant of the device together with the gate oxide capacitance It will be clear that if the film resistivity is a function of environmental conditions such as humidity, the time-delay measurement is actually the measurement of this external parameter Various polymer films are used as well as sputtered glass film for humıdity, gas and smoke detection In fact the advantage of the MOS basis of this device is the possibility of measuring the resistance of thin films with a very high resistivity, which would be very difficult with more conventional measuring techniques

However, the actual sensing mechanism has nothing to do with the FET construction, because it is completely controlled by the chemical or physical affinity of the resistive film to environmental parameters On the other hand, the technology of coating the transistor with a resistive film is wholly compatible with MOSFET technology

Device construction and operation are schematically represented in Fig 6

\section{The PRESSFET}

As mentioned above, in the CFT construction use is made of a lateral shift of the gate metal, whlle the resistive film is a coupling element with the actual gate area Of course this shift can also be provided in a direction perpendicular to the transistor If, in contrast to the CFT, the material between the elevated gate and the gate oxide is a dielectric, the MOS transistor will electrically still obey the equations mentioned in Section 2 In eqn (1) the notation $C_{o x}$ for the oxide capacitance has now to be replaced by that of the equivalent capacitance $C_{\mathrm{eq}}$ of the dielectric sandwich on top 


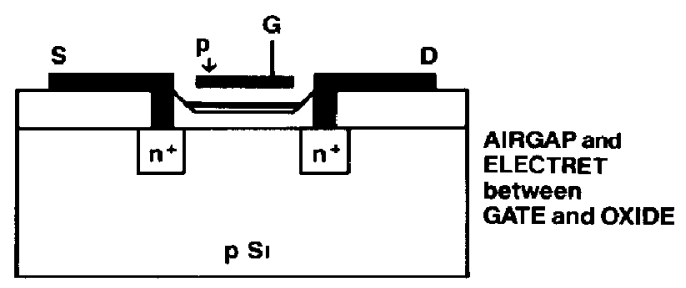

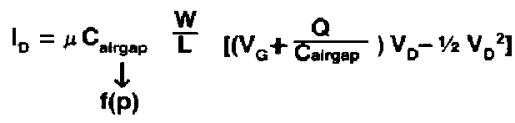

$$
\begin{aligned}
& \frac{d l_{D}}{d C_{\text {airgap }}}=\mu \frac{W}{L}\left(V_{G} V_{D}-1 / 2 V_{D}{ }^{2}\right) \\
& { }_{200} \text { Volt from electret }
\end{aligned}
$$

Fig 7 Schematic representation and basic operation of the PRESSFET

of the gate $C_{\mathrm{eq}}$ may be a function of the external environment, for instance as the lifted gate bends as a function of pressure when the space between the original gate oxide and the lifted gate is vacuum or simply air

Because realistic values of an arr gap thickness are of the order of microns, the resulting capacitance is

$C_{\text {eq }} \approx C_{\text {a1rgap }} \ll C_{\text {ox }}$

This means that eqns (1), (2) and (3) can now be approximated by

$I_{\mathrm{D}}=\frac{\mu C_{\text {arrgap }} W}{L}\left[\left(V_{\mathrm{G}}-2 \phi_{\mathrm{f}}-\phi_{\mathrm{ms}}+\frac{Q_{\text {tot }}}{C_{\text {airgap }}}\right) V_{\mathrm{D}}-\frac{1}{2} V_{\mathrm{D}}^{2}\right]$

where $Q_{\text {tot }}=Q_{\mathrm{B}}+Q_{1 \mathrm{t}}+Q_{\mathrm{f}}$ is thus

The sensitivity of the device for a variation of the aurgap capacitance

$\frac{\mathrm{d} I_{\mathrm{D}}}{\mathrm{d} C_{\text {a1rgap }}}=\mu \frac{W}{L}\left[\left(V_{\mathrm{G}}-2 \phi_{\mathrm{f}}-\phi_{\mathrm{ms}}\right) V_{\mathrm{D}}-\frac{1}{2} V_{\mathrm{D}}^{2}\right]$

This sensitivity can be increased by increasing the applied voltages, especially $V_{G}$, simply because a higher voltage on a capacitor implies a higher charge Therefore voltages of the order of several hundred volts are applied to condenser microphones These values are, however, very unrealistic to be applied to sensors from an external source This problem is solved for condenser microphones by incorporating an additional fixed charge in the capacitive structure, resulting in the well-known electret microphones This electret concept can also be applied to pressure-sensitive devices as mentioned above This will lead to the development of a PRESSFET, which can be used in a catheter-tip construction for the measurement of blood pressure and as a solid-state configuration of the electret microphone for application in hearing alds [12] In both cases the dielectric sandwich of the MOSFET 
gate also contains, besides the sulicon dioxide and an aurgap, a layer of Teflon containing the fixed charge, which results in an equivalent gate-source voltage $V_{G}$ of the order of several hundred volts The PRESSFET concept is schematically represented in Fig 7

A similar sandwich approach is the use of a piezoelectric polymer, such as $\mathrm{PVF}_{2}$, as a layer between the metal gate and the oxide of a MOSFET In this case no aurgap is necessary, because an acoustic pressure on the piezoelectric layer directly induces an input voltage for the attached MOSFET This type of acoustically sensitive transducer is especially developed for use as arrays in medical ultrasonic imaging systems [13]

\section{0 'Ihe ISFET}

In the terms of this paper, the already widely-known Ion Sensitive Field Effect Transistor (ISFET) can be seen as a special type of MOSFET with a gate at a certain distance [14] In this case no dielectric sandwich is inserted between the gate metal and the silicon dioxide as is done with the PRESSFET, but instead a conducting electrolyte and a half cell, originating from the reference electrode The metal part of this reference electrode can be seen as the actual gate of the FET system, to which the original equation of the MOSFET can still be applied (eqn (1))

However, in the case of an ISFET the equation of the flatband voltage (eqn (3)) contains some additional terms, which describe the ion sensitivity [15]

$V_{\mathrm{FB}}=E_{\mathrm{ref}}-\psi_{0}+\chi^{\mathrm{sol}}-\frac{\Phi_{\mathrm{si}}}{q}-\frac{Q_{1 \mathrm{t}}+Q_{\mathrm{f}}}{C_{\mathrm{ox}}}$

This differs from eqn (3) in that $E_{\text {ref }}$, the reference electrode potential relative to vacuum, now contains the metal work function $\Phi_{M} / q$, whlle the additional term $\left(-\psi_{0}+\chi^{\text {sol }}\right)$ describes the interfacial potential at the electrolyte/silicon dioxide interface The factor $\chi^{\text {sol }}$ is the surface dipole moment of the solution, which can be considered to be constant, while $\psi_{0}$ is the actual driving force of the ISFET, related to the solution $\mathrm{pH}$ with respect to the $\mathrm{pH}$ that does not result in a net surface charge, $\mathrm{pH}_{\mathrm{pzc}}$ (point of zero charge)

$\psi_{0}=2303 \frac{k T}{q}\left(\frac{\beta}{\beta+1}\right)\left(\mathrm{pH}_{\mathrm{pzc}}-\mathrm{pH}\right)$

for the condition that $\beta \gg q \psi_{0} / k T$ The parameter $\beta$ reflects the chemical sensitivity of the outer oxide surface, depending mainly on the site density $N_{\mathrm{s}}$ of the hydroxyl groups, and the surface reactivity characterized by the equilibrium constants $K_{\mathrm{a}}$ and $K_{\mathrm{b}}$ of the acidic and basic surface reactions

$\beta=\frac{2 q^{2} N_{\mathrm{s}}\left(K_{\mathrm{a}} K_{\mathrm{b}}\right)^{1 / 2}}{k T C_{\mathrm{DL}}}$ 


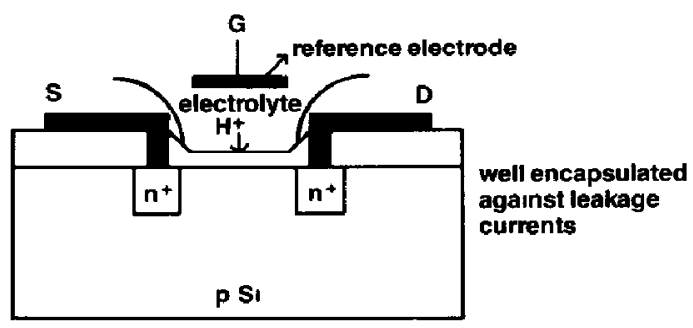

$$
\begin{aligned}
& I_{D}=\mu C_{o x} \frac{W}{L}\left[\left(V_{G}-V_{T}\right) V_{D}-1 / 2 V_{D}{ }^{2}\right] \\
& \downarrow v_{F B}-\frac{Q_{B}}{C O X}+2 \phi_{f} \\
& \begin{aligned}
\stackrel{\downarrow}{=} \mathrm{E}_{\text {ref }} & -\Psi_{0}+x^{301}-\frac{\Phi_{S_{1}}}{q}-\frac{Q_{1 t+} Q_{f}}{C_{o x}} \\
& \rfloor_{=23} \frac{\mathrm{kT}}{q} \frac{\beta}{\beta+1}\left(\mathrm{pH}_{\mathrm{pzc}}-\mathrm{pH}\right)
\end{aligned}
\end{aligned}
$$

Fig 8 Schematic representation and basic operation of the ISFFT

where $C_{\mathrm{DL}}$ is the double-layer capacitance, which can in practice be approximated as being constant

Thus the ISFET can be seen as a normal MOSFET with the oxide replaced by an oxide-electrolyte system, resulting in a $\mathrm{pH}$-dependent threshold voltage The applied voltages and the electrical sensitivity of the device are of the same order of magnitude as in normal MOSFETs

The selectivity and chemical sensitivity of the device are completely controlled by the properties of the insulator/electrolyte interface It has been shown that for $\mathrm{pH}$ sensors inorganic gate materials other than $\mathrm{SiO}_{2}$ are favourable $\mathrm{Al}_{2} \mathrm{O}_{3}$ and $\mathrm{Ta}_{2} \mathrm{O}_{5}$ especially appear to be the best for $\mathrm{pH}$ measurement, because the parameter $\beta$ (eqn (11)) is much higher for these materials In practice, the layers of $\mathrm{Al}_{2} \mathrm{O}_{3}$ and $\mathrm{Ta}_{2} \mathrm{O}_{5}$ are simply deposited on top of the first layer of $\mathrm{SiO}_{2}$ by means of chemical vapour deposition (CVD), as is usual in other MOS devices with sandwiched dielectric layers

So far the fabrication of ISFETs is completely similar to that of MOSFETs, only the gate metallization being omitted The usual inorganic gate insulators appear to possess the required $\mathrm{pH}$-sensitive properties, fairly well matched with the $\mathrm{pH}$ range, sensitivity and selectivity of interest for common chemical and biomedical applications This is a very fortunate coincidence The ISFET is schematically represented in Fig 8

As soon as different properties are required for special purposes, the insulator surface has to be modified This can be done by ion implantation If one wants to be compatible with IC technology, or else by chemical treatment of the surface A good understanding of the site-dissociation model, on which eqns (10) and (11) are based, also gives an answer to the question about what should and can be done with the surface hydroxyl groups of the original inorganic gate material One can increase or dimmish the number of acidic and or basic groups by means of adequate chemical treatment, giving a predictable shift of the $\mathrm{pH}_{\mathrm{pzc}}$ and $\psi_{\mathrm{o}} / \mathrm{pH}$ relation $[16,17]$ 

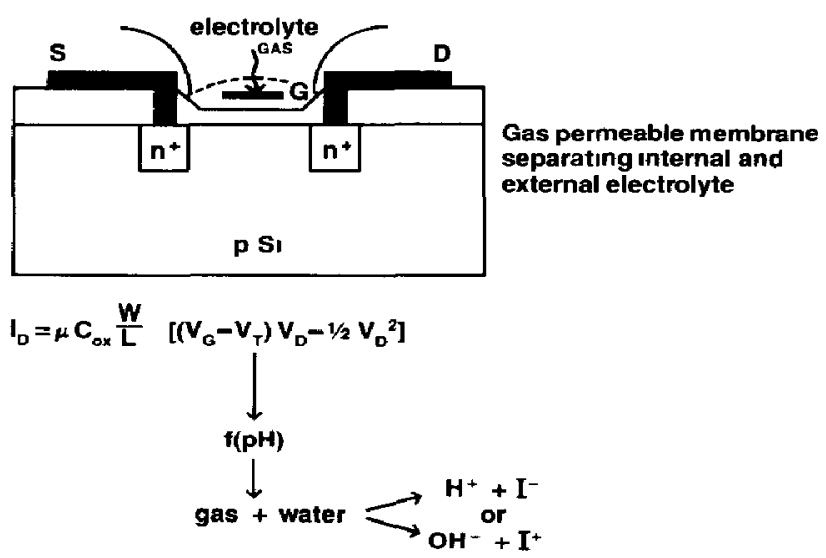

Fig 9 Schematic representation and basic operation of the dissolved-gas FET

It will be clear that the ISFET as a $\mathrm{pH}$-sensitive device can also be used indirectly for measuring a chemical quantity that controls the $\mathrm{pH}$ in a chamber containing the ISFET and reference electrode and separated from the environment by a membrane Measurements of the partial pressures of $\mathrm{CO}_{2}$ and $\mathrm{NH}_{3}$ can be obtained in this way [18], which is simular to the more macroscopic $\mathrm{CO}_{2}$ and $\mathrm{NH}_{3}$ electrodes constructed on the base of glass membrane electrodes However, sllicon micro-machining combined with ISFET technology provides a more direct method of constructing complete structures of such a sensor for dissolved gases

Basically these devices are still ISFE'Ts, but are directly coupled with a chemical convertor, represented as shown in Fig 9

\section{The REFET}

An extreme case of surface modification, as mentioned in the previous section, is the realization of a condition where the $\mathrm{pH}$ sensitivity is so far diminished that one can speak of $\mathrm{pH}$ insensitivity, at least in a certain $\mathrm{pH}$ range A chemical treatment that will result in such a device is extremely interesting, because it can be used as a reference ISFET (a REFET according to the literature), the development of which could be the solution for the reference electrode problem The REFET is shown schematically in Fig 10

The ISFET and REFET should be applied in a differential circuit, in which case a platinum electrode can serve as the connection with the electrolyte, because now the interference of the electrode potential will be common to the ISFET and REFET and will thus not be measured

Up to now no REFETs have been realized with only a chemical treatment of the surface Present REFETs have gate insulators that are made $\mathrm{pH}$ insensitive by means of an additional coating such as Parylene, which makes the surface hydroxyl groups less active simply by coverage [16] It appears, 

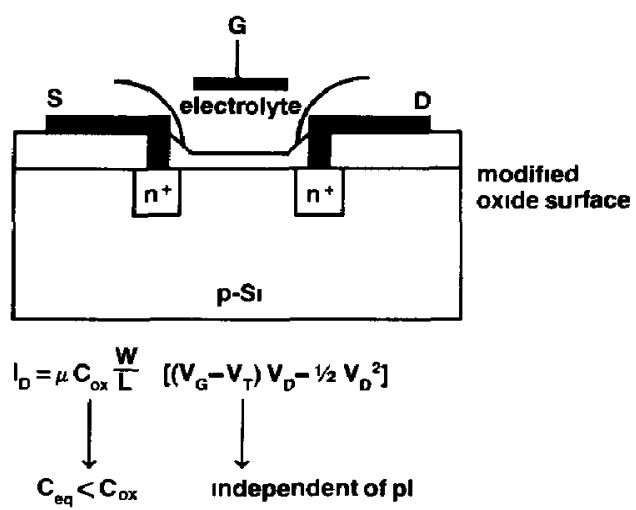

Fig 10 Schematic representation and basic operation of the REFET

however, that in this case the $\mathrm{pH}$ sensitivity cannot be completely removed Future research will probably lead to chemical surface modification combined with coverage, resulting in a complete masking of hydroxyl or similar surface groups

Note that such a REFET is in fact again a pure field effect device The field inside the insulator is only a function of an applied potential

\section{The CHEMFET}

In Section 10, the notation ISFET was more or less reserved for those devices whose inorganic gate materials are well known in MOS technology and fortunately also have ion-sensitive properties, which appear to be limited to $\mathrm{pH}$ sensitivity

In order to be able to develop ion sensors that are selectively sensitive for other 1ons, no materials are known that can be compatibly produced with MOS technology What is known from the development of conventional ion-sensitive electrodes (ISEs) is that partıcular organic materials containing a certain amount of plastisizer can act as a substrate for a variety of conexchange materials These electrodes are called solid-state membrane electrodes Analogously with this technology, ISFETs are also being coated with these organic membranes which are made sensitive for specific ions These coated ISFETs are commonly called CHEMFETs, a combination of a conductive chemical sensitive layer and the potential-sensitive FET structure A well-known example is the potassium-sensitive CHEMFET, which is coated with PVC containing valınomycin as ion exchanger [19]

Because the membrane has conducting properties, the thickness of the layer is less critical than in ISFETs with additional insulating layers The latter layers are always made by means of MOS technology, where thickness can be very accurately controlled, even with very thin layers, while the CHEMFET top layer can be provided by a more simple method, namely dipcoating in a suspension of the membrane material However, the 

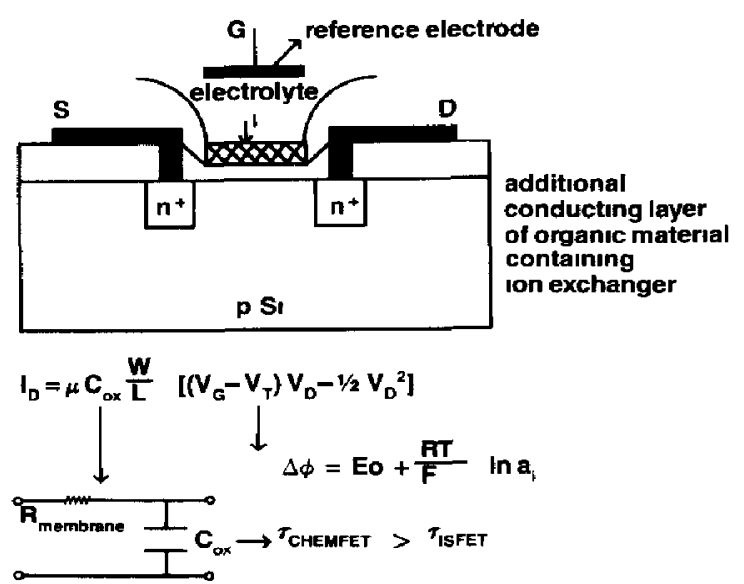

Fig 11 Schematic representation and basic operation of the CHEMFET

time constant of the CHEMFET slows down with respect to that of the original ISFET due to the resistance of the membrane A CHEMFET is schematically shown in Fig 11

The most critical point of the CHEMFET construction is the attachment of the membrane to the underlyung inorganic gate material With dipcoating one has to rely on a physical bond, which is of course not one of the best possibulities Indeed in practice CHEMFETs often suffer from membrane loosening as they are in contact with water This is a serious drawback with respect to their life-time

In the future one can expect an approach similar to the one described for a REFET, where in this case the chemical modification of the oxide surface will not only protect this surface against protonic reactions but will also lead to chemical bonding of an ion-sensitive membrane In this respect a crown-ether layer is a very promising perspective

\section{The ENFET}

The ENFET is in principle a CHEMFET, but the membrane is in this case a gel, containing immobllized enzymes These enzymes control very specific chemical reactions, resulting in chemical products that can be measured by the underlying ISFET [20]

For example, urea is measured by means of the enzyme urease, resulting in the production of ammonium and bicarbonate ions according to

$\left(\mathrm{NH}_{2}\right)_{2} \mathrm{CO}+2 \mathrm{H}_{2} \mathrm{O}+\mathrm{H}^{+} \stackrel{\text { urease }}{\longrightarrow} 2 \mathrm{NH}_{4}{ }^{+}+\mathrm{HCO}_{3}^{-}$

The corresponding $\mathrm{pH}$ change at the ISFET surface can be related to the original urea conc entration This is symbolized in Fig 12

Although Figs 11 and 12 suggest a similar operation of the CHEMFET and ENFET, it should be noted that this is not the case A CHEMFET 


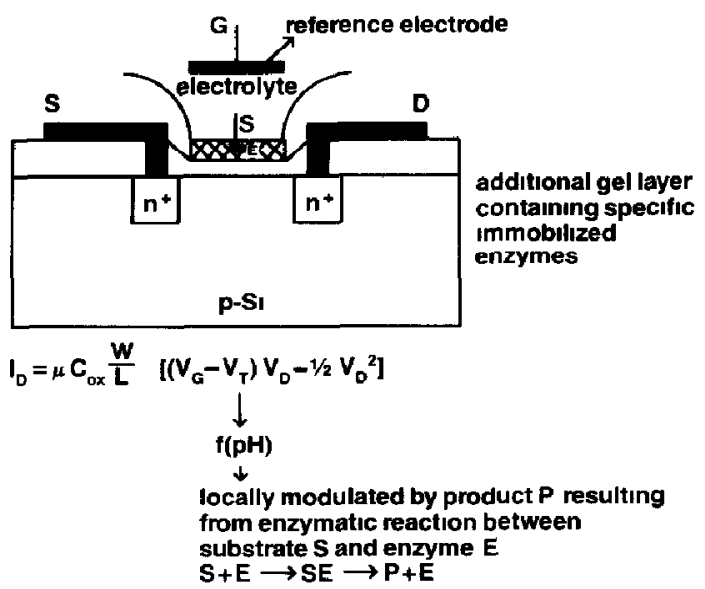

Fig 12 Schematic representation and basic operation of the ENFET

measures the diffusion potential at the interface between the added layer and the liquid as a function of ion activity $l$ in the liquid An ENFET measures, on the contrary, not an interfacial potential but a local $\mathrm{pH}$ change in the bulk of the added gel layer as a function of an undiffused substrate $S$ However, the measured effect is also a function of the $\mathrm{pH}$ and buffer capacity of the liquid, which is one of the disadvantages of this type of enzyme sensor

The development of ENFETs is faced with the same problem of membrane fixation as discussed in the previous section An even more serious problem of these devices is related to the nature of the enzymes, which will certainly degenerate relatively fast under these immobllized curcumstances

As with the CHEMFET, the problems with the ENFET do not originate from the particular sensing principle, $l e$, the measurement of an electric field in an insulator adjacent to a semiconductor This concept is clear and still worthwhle applying The lifetıme improvement of these devices depends more or less on the synthesis of new materials that can be chemically bonded to an inorganic insulator and can contain a fixed and stable amount of 1on- or molecule-sensitive materials

As in the case of $\mathrm{CO}_{2}$ and $\mathrm{NH}_{3}$ electrodes, the ISFET in the ENFET mode is still in use as a $\mathrm{pH}$ sensor, but directly coupled with a chemical convertor

A combination of enzymes and a $\mathrm{CO}_{2}$ or $\mathrm{NH}_{3}$ ISFET (Fig 9) is also a possibility, as well as a combination of enzymes and a GASFET (Fig 2), where a gaseous species such as $\mathrm{NH}_{3}$, evolved in an enzymatic reaction, is detected directly [7]

\section{The IMFET}

As the ISFET has proved to be a device that is very sensitive for any electrical interaction at the surface, it may also be useful for measuring 


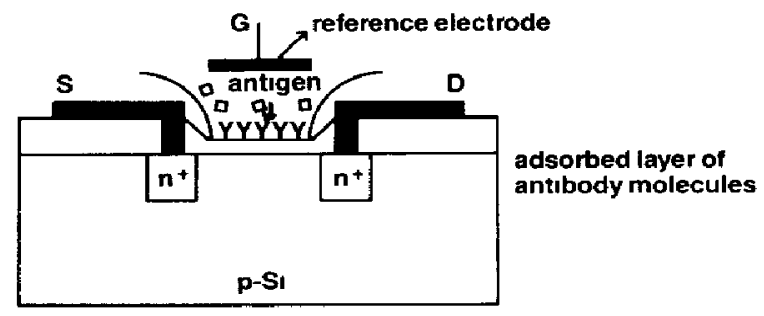

$$
I_{D}=\mu C_{o x} \frac{W}{L}\left[\left(V_{G}-V_{T}\right) V_{D}-1 / 2 V_{D}{ }^{2}\right]
$$

Fig 13 Schematic representation and basıc operation of the IMFET

electrical effects caused by immunological reactions at the surface Therefore a layer of antibody or antigen molecules has to be absorbed or covalently bonded to the ISFET surface, as with the ELISA (Enzyme Linked Immunosorbent Assay) technique [21] It is expected that a reaction with the specific antigen or antıbody will result in an equivalent threshold voltage change, but this has still to be proved under realistic conditions In particular the existence of water molecules and salt ions in the immunologically-active layer will negatively influence the sensitivity of the device [22] Much more research will have to be done in order to realize stable and accurate measurements with an immuno-FET (IMFET), which is schematically represented in Fig 13

\section{The BIOFET}

The ENFET and IMFET make use of biologically active substances directly coupled with an ISFET

The layer of immobllzed enzymes on top of the ENFET can be seen as a biochemical converter of substrate concentration to local $\mathrm{pH}$ changes, or in other words, it operates as a biologically-controlled pH actuator With the IMFET use is made of a direct conversion of a biochemical reaction to an electrical signal, measured by the ISFET

In general, it will be clear that any biochemical reaction resulting in chemical or electrical changes can be measured by means of a coupled ISFET

The appearance and construction of these devices will be similar to those that measure dissolved gases In that case, a chemical converter was an implicit part of the sensor chip In the case of a BIOFET, this chemical converter is replaced by a biochemical convertor or reactor

Such ISFET-coupled bioreactors may contain all kinds of biologically active species, even including bacteria, whole cell and living tissue [23] The reactors can be seen as biological receptors, which can very selectively recognize chemical and bıochemical substances 

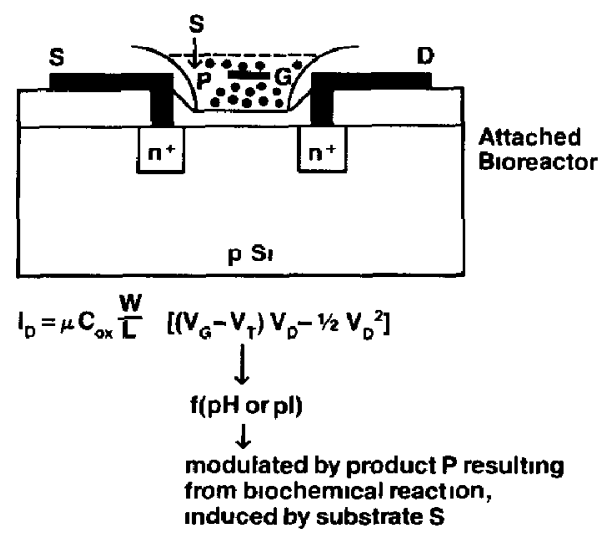

Fig 14 Schematic representation and basic operation of the BIOFET

In order to develop such BIOFETs (see Figure 14), great advantage can be taken of the progress that has been made in biotechnology research, where biological activity is also used functionally All ISFETs have to do is to measure the product If the original substance to be measured reacts with a reagent through a cataly tic cycling with a multiplication factor, a relatively large amount of product is generated, which means that the sensor contains an inherent chemical amplification beside its electronic amplification This will increase the sensitivity in a similar way to that known from biological transducers It should also be noted that the very thin layers of active material found in some bioreceptors can easily be imitated by means of the ISFET concept with added thin layer and membrane constructions

The development of BIOFETs has however, hardly started, but will certainly be an important research topic in the near future

\section{Conclusion}

It can be concluded that all the sensors described in this paper are FET-based, which means that the field-dependent silicon inversion layer is measured Note that the sensors are considered as modified MOSFETs, from which another parameter rather than a change in the gate-source voltage is responsible for the modulation of the drain current This is due to the inherent integration of the sensor and the electronic functional parts of the total device

Of course in practical cases one can also separate the two distinguishable functions Many examples can be found in the literature concerning sensors constructed in slicon and integrated with an MOSFET or even complete CMOS circuitry In those cases the MOSFET function is not modified, but the MOSFET is simply driven by the output signal of the sensor

Seen from an electronic point of view, all FET-based sensors described in this paper behave in a simular way, which means that they can be part of an electronic measuring circuit in a rather standard configuration This 
makes the development of FET-based sensors very interesting, as well as the fact that basically the sensor technology is also the same This is of great importance, especially for the development of multı-sensors

\section{Acknowledgements}

All members of the blosensor group are gratefully acknowledged for their critical remarks and enthusiasm in proving the valıdity of FET-based sensors in some of the sensor developments described here

\section{References}

1 J E Lilienfeld, US Patent 1745175 (1930)

2 W Shockley and G L Pearson, Modulation of conductance of thin films of semıconductors by surface charges, Phys Rev, 74 (1948) 232

$3 \mathrm{H} \quad \mathrm{K} \quad \mathrm{J}$ Ihantola and $\mathrm{J} \mathrm{L}$ Moll, Design theory of a surface field-effect transistor, Solid-State Electron, 7 (1964) 423

4 L Forbes, L L Wittmer and $\mathrm{K}$ W Loh, Characteristics of the Indium-doped infrared sensing MOSFET, IEEE Trans Electron Devices, ED-23 (1976) 1272

5 I Lundstrom, M S Shivaraman, C S Svenson and L Lundkvist, Hydrogen-sensitive MOS field effect transistor, Appl Phys Lett, 26 (1975) 55 - 57

6 D Krel, K Dobos and G Zimmer, An integrated CO-sensitive MOS transistor, Sensors and Actuators, 1 (1983) 169

7 F Winquist, I Lundstrom and B Danielsson, Biosensors based on ammonia sensitive MOS structures, Digest of Technical Papers, Transducers' 85, IEEE Catalog no $8 S C H 2127-9,1985, \mathrm{p} 162$

$8 \mathrm{~B}$ Thorstensen, Field effect studies of gas adsorption on oxidized silicon surfaces, Thesis, University of Trondheim, Norway, 1981, report no STF 44A 81118

9 P F Cox, Environment monitoring device and system, US Patent 3831432 (1974)

$10 \mathrm{M}$ Stenberg and B I Dahlenback, Surface accessible FET for gas sensing, Sensors and Actuators, 4 (1983) 273

11 S D Senturia, $C M$ Secken and $J$ A Wishnenski, The charge flow transistor a new MOS device Appl Phys Lett, 30 (1977) 106

$12 \mathrm{~J} A$ Voorthuyzen and $\mathrm{P}$ Bergveld, Theoretical considerations in the design of integrated semiconductor sensors applying electrets, IEEE Trans Electron Devices, $E D-32$ ( 7) (1985) 1185

$13 \mathrm{R} G$ Swartz and J D Plummer, Integrated silicon-PVF 2 acoustic transducer arrays, IEEE Trans Electron Devices, ED-26 (12) (1979) 1921

$14 \mathrm{P}$ Bergveld and $\mathrm{N} F$ de Rooy, The history of chemically sensitive semiconductor devices, Sensors and Actuators, 1 (1981) 5

15 L Bousse, N F de Roolj and P Bergveld, Operation of chemically sensitive fieldeffect sensors as a function of the insulator-electrolyte interface, IEEE Trans Electron Devices, ED-3O (1983) 1263

$16 \mathrm{~T}$ Matsuo and $\mathrm{H}$ Nakajıma, Characteristics of reference electrodes using a polymer gate ISFET, Sensors and Actuators, 5 (1984) 293

17 A van den Berg, $P$ Bergveld, $D N$ Reinhout and $E$ J $R$ Sudholter, Sensitivity control of ISFETs by chemical surface modification, Sensors and Actuators, 8 (1985) 129

$18 \mathrm{~K}$ Ahimada, M Yano et al Application of catheter-tip ISFET for continuous in vivo measurement, Med Biol Fing Comp, 18 (1980) 741 
19 A Sibbald, Chemical-sensitive field effect transistors, IEE Proc, 130 (1983) 233 (130 references)

20 Y Miyahara, F Matsu and T Mirizumi, Micro enzyme sensors using semiconductor and enzyme-ımmobilızation techniques, in $T$ Seiyama, S Suzukı, K Fuekı and J Shiokawa (eds), Chemical Sensors, Analytical Chemistry Symposia Series, Vol 17, Elsevier, Amsterdam, 1983, pp 501 - 506

21 E Engvall and P Periman, Enzyme-lınked immunosorbent assay (ELISA) Quantıtative assay of immunoglobulın G, Immunochemistry, 8 (1971) 871

$22 \mathrm{~J} \mathrm{~F}$ Schenck, Technical difficulties remaining to the application of ISFET devices In Theory, Destgn and Biomedical Applications of Solzd State Chemical Sensors, C R C Press, Boca Raton, FL, 1978, p 165

23 In T Selyama, S Suzukı, K Fuekı and J Shıokawa (eds), Chemical Sensors, Analytical Chemistry Symposia Series, Vol 17, Elsevier, Amsterdam, 1983, chapter on Biosensors 